\title{
Grado de satisfacción, utilidad y validez de la evaluación con rúbricas electrónicas durante el prácticum.
}

\section{Degree of the satisfaction, utility and validity of electronic rubrics evaluation during the practicum.}

\author{
Ana Belén Pérez Torregrosa \\ Universidad de Granada \\ anabpt91@correo.ugr.es
}

María Asunción Romero López

Universidad de Granada romerol@ugr.es

Pilar Ibáñez Cubillas

Universidad de Granada pcubillas@ugr.es

\section{Dra. María Jesús Gallego Arrufat Universidad de Granada mgallego@ugr.es}

Pérez-Torregrosa, A.B., Romero-López, M.A., Ibáñez-Cubillas, P. \& Gallego-Arrufat, M.J. (2017). Grado de satisfacción, utilidad y validez de la evaluación con rúbricas electrónicas durante el prácticum. Revista Prácticum, 2(1), 60-79. 


\section{Resumen}

El propósito de este estudio es conocer las opiniones de los futuros docentes ante el empleo de las e-rúbricas durante el prácticum. Con el fin de conocer y comprender sus opiniones analizamos las variables de grado de satisfacción, utilidad y validez, así como el potencial grado de recomendación a otros tras el uso de dicho instrumento. Los datos se obtienen de un cuestionario ad hoc diseñado para este fin. La muestra está formada por 29 estudiantes del Prácticum I (2016/2017) de los Grados de Educación Infantil y Primaria de la Universidad de Granada (España). Se seleccionan dos grupos de prácticum: uno experimental y otro control, para comprobar las diferencias entre el grupo que empleó la e-rúbrica durante su período de prácticas y el que no. Tras los datos obtenidos, podemos concluir que los futuros docentes han valorado positivamente el uso de la e-rúbrica, pues consideran que les ha ayudado a reflexionar sobre sus habilidades adquiridas y el proceso desarrollado. Es necesario continuar investigando ya que se ha tratado de un estudio exploratorio. Las rúbricas electrónicas pueden considerarse de utilidad para mejorar los procesos de evaluación del período de prácticas.

\section{Abstract}

The purpose of this study is knowing what future teachers think about the use of electronic rubrics (e-rubrics) during the practicums. We analyse the variables of degree of the satisfaction, utility and validity. Besides the degree of the potential recommendation to others after the use of that instrument. The data is obtained from an ad hoc questionnaire designed for this propose. The sample comprises 29 students of Practicum I (2016/2017) of the degree of Education from the University of Granada (Spain). Two groups of practicums have been selected (one experimental and one control) to check the difference between the group who used e-rubric during the practicum and the group who didn't use it. Having analysed the data we can conclude that future teachers have positively appraised the use of the e-rubric. They consider that it has helped them to rethink about the skills they have acquired. It becomes necessary keep on researching since it has been an exploratory study. E-rubrics can be considered useful to improve the evaluation processes of the practicum period. 


\section{Palabras claves}

Satisfacción, Evaluación, Prácticum, Rúbricas, Estudiantes de magisterio, Evaluación con rúbricas electrónicas

\section{Keywords}

Satisfaction, Evaluation, Practicums, Scoring Rubrics, Student teachers, Electronic rubric-based assessment

\section{Introducción}

En la actualidad se han comenzado a incorporar las Tecnologías de la Información y Comunicación (TIC) en educación en general, y también en el prácticum de los futuros docentes. Las TIC proporcionan nuevas posibilidades para la evaluación durante este período, aunque su introducción implica la adquisición de nuevos conocimientos y habilidades, y la influencia de las actitudes de los futuros docentes en el empleo de las nuevas herramientas. De forma paralela a la evaluación de competencias en la reforma del EEES, la rúbrica se ha comenzado a popularizar en educación superior, en evaluación de actividades, trabajos, y prácticas. Por eso, junto con los ecuestionarios y eportafolios, se están comenzando a emplear en la evaluación de los estudiantes en su período de prácticas las rúbricas electrónicas (e-rúbricas). "Con frecuencia los estudios no distinguen la rúbrica de la e-rúbrica. Sólo en ocasiones si la modalidad (online, blended o presencial), el soporte y su disponibilidad diacrónica difieren. Pero el instrumento es idéntico y la prospectiva de investigación también" (Gallego-Arrufat \& RaposoRivas, 2014, p.200).

Encontramos numerosos estudios sobre la influencia que ejerce el uso de las rúbricas en el aprendizaje y evaluación de los estudiantes, así como su impacto en la calidad de las calificaciones (Andrade \& Du, 2005; Jonsson \& Svingby, 2007; Andrade, Du \& Mycek, 2010; Gallego-Arrufat \& Raposo-Rivas, 2014). Las rúbricas son empleadas en educación para evaluar de una gran variedad de constructos. Utilizadas como parte de un enfoque de evaluación centrada en el estudiante, las rúbricas tienen el potencial de ayudarles a entender los objetivos de su aprendizaje y los estándares de calidad para una tarea en particular, además de realizar juicios fiables sobre su propio trabajo que pueden informar sobre su revisión y mejora (Reddy \& Andrade, 2010). Por lo tanto, este estudio considera que las e-rúbricas son una herramienta esencial para 
apoyar el aprendizaje de los futuros docentes, así como para facilitar una evaluación más eficiente de su prácticum. Pero la simple implementación de rúbricas no garantiza una evaluación efectiva.

En este estudio empleamos las e-rúbricas para fomentar dos aspectos de la evaluación formativa, concretamente la autoevaluación y la co-evaluación.

La evaluación colaborativa (co-evaluación) o evaluación entre pares se centra en el estudiante y se describe como un proceso para hacer juicios críticos de los compañeros (Fry, 1990). Más concretamente, como un acuerdo para que los compañeros consideren el nivel, el valor, la calidad o el éxito de los productos o resultados de aprendizaje de otros de estado similar (Topping, Smith, Swanson \& Elliot, 2000). También les da la oportunidad de comparar su trabajo con el de un compañero, proceso que requiere una mayor consciencia metacognitiva y contribuye al desarrollo de habilidades de autoevaluación (Topping et al. 2000). Existen investigaciones que apoyan la aplicación de rúbricas y e-rúbricas para este tipo de evaluación (Prins, Sluijsmans, Kirschner \& Strijbos, 2005; Reddy \& Andrade, 2010; Panadero, Romero \& Strijbos, 2013), pero los estudios centrados en la formación inicial de los docentes y la adquisición de competencias profesionales son más escasos (Cebrián de la Serna, Serrano Angulo \& Ruiz Torres, 2014). Así, este estudio se analiza la aplicación e influencia de e-rúbricas en dicho contexto.

La autoevaluación va a permitir a los futuros docentes concentrarse en los aspectos más importantes que necesitan mejorar y construir las habilidades necesarias para cualquier área de su trabajo (Boud, Lawson \& Thompson, 2013). Según Panadero \& Romero (2014), su relevancia se manifiesta por la gran cantidad de evidencias empíricas que encontramos sobre su utilidad y eficacia acumulada en las últimas tres décadas. Definen la autoevaluación como:

"la valoración cualitativa del proceso de aprendizaje y del producto final obtenido a partir de unos criterios de evaluación. Esta definición incluye no sólo la valoración del resultado final, sino también del proceso a través del cual se llega a éste. Nuestra definición incluye un aspecto clave: los criterios de evaluación". (Panadero \& Romero, 2014, p.174)

Establecer unos criterios para lograr una efectiva autoevaluación es fundamental ya que, haciéndose explícitos, el alumnado estará más seguro de cómo evaluar y si lo están haciendo correctamente o no (Panadero \& Romero, 2014). Para la autoevaluación del alumnado se emplearán e-rúbricas, que contienen 
estos criterios y estándares de calidad. Ambas características son esenciales para que se lleve a cabo una correcta autoevaluación. Los futuros maestros deben ser conscientes de la importancia de la autoevaluación y de la mejora que puede provocar en su crecimiento profesional.

Los tres constructos que se analizan en el presente estudio son: el grado de satisfacción, la utilidad y la validez percibida por los estudiantes.

El primero es la satisfacción, concepto derivado de la mercadotecnia, se ha convertido en un factor cada vez más importante en una sociedad muy competitiva. Por ello, organismos e instituciones tratan de dar respuesta a las necesidades de sus clientes, como medio para ofrecer calidad y excelencia de sus productos o servicios. En las últimas décadas se ha ampliado su utilización en diversos ámbitos: satisfacción laboral; satisfacción del usuario; satisfacción de utilización o, con carácter genérico; la satisfacción personal. Concretamente en el ámbito de la educación y formación, se atiende a la satisfacción como un elemento desde el cual medir la calidad de los servicios o el rendimiento de estudiantes y profesorado. De ahí, la presencia de una línea de investigación centrada en la medición de la satisfacción de estudiantes universitarios como percepción de calidad (Perrone \& Propper, 2007).

En el ámbito de este estudio, es válida la definición, como descriptor, del Thesaurus de ERIC. La satisfacción es entendida como "evaluación de los estudiantes ante una experiencia, valorando en qué medida se ajusta a sus necesidades o expectativas". Desde este concepto, dado que las e-rúbricas pueden considerarse un producto puesto a disposición de los estudiantes, es necesario conocer el grado de satisfacción que presentan tras su uso, con el fin de proporcionar un mayor beneficio en el proceso de aprendizaje y promover medidas que atiendan a las necesidades de los estudiantes ante esta herramienta de aprendizaje.

Existen investigaciones en las que los estudiantes ponen en relieve la satisfacción ante el uso de e-rúbricas empleadas en determinadas situaciones, a nivel internacional (Atkinson \& Lim, 2013; Auvinen, 2011) y nacional (Serrano Angulo \& Cebrián Robles, 2014; Raposo-Rivas \& Gallego-Arrufat, 2016). Auvinen (2011) obtiene evidencias de la eficacia de las e-rúbricas como herramienta de evaluación y la satisfacción de los estudiantes ante esta herramienta. También Raposo-Rivas \& Gallego-Arrufat (2016) concluyen que los estudiantes de magisterio usuarios de e-rúbricas muestran una alta satisfacción en su empleo, no sólo como 
revista

herramienta de evaluación, empleada en el estudio para autoevaluación y evaluación de pares, sino también de aprendizaje.

El segundo constructo analizado es la utilidad. Con el desarrollo de las TIC han surgido diferentes modelos que integran la utilidad de la tecnología. Uno de ellos es el modelo de aceptación tecnológica (Technology Acceptance Model, TAM), en el que la utilidad se entiende como "el grado en que una persona cree que, empleando un determinado sistema, mejorará su rendimiento en el trabajo" (Davis, 1989, p. 320). Este ha demostrado ser un modelo teórico que ayuda a explicar y predecir el comportamiento del usuario ante cualquier tecnología de la información (Legris, Ingham \& Collerette, 2003) y ha sido usado en numerosas investigaciones empíricas tanto a nivel internacional (Selim, 2003; Park, 2009; Teo, Lee, Chai \& Wong, 2009) como nacional (Padilla Meléndez \& Garrido Moreno, 2006; Casaló, Flavián \& Guinalíu, 2012; Sánchez, Martín \& Villarejo, 2016). La utilidad de las rúbricas ha sido comprobada en varios estudios tanto desde una perspectiva formativa como sumativa (Hoepfl, 2007). En la presente investigación se entiende utilidad como el grado en que los futuros docentes creen que el uso de las e-rúbricas les ayudará en su autoevaluación y a reflexionar sobre las habilidades que han adquirido durante su prácticum.

El tercer constructo es la validez percibida por los estudiantes, definida en este estudio como la opinión acerca de la capacidad de la erúbrica para evaluar de forma significativa y adecuada las competencias adquiridas en sus prácticas. La medida en que una erúbrica arroja evidencias empíricas del grado en que las competencias han sido adquiridas o se encuentran en proceso de adquisición legitima no sólo el empleo de la erúbrica sino también el resultado de su aplicación.

Se han utilizado varias estrategias para analizar la validez de una rúbrica. Algunos autores recomiendan mejorar la validez del contenido de la rúbrica, involucrando a los profesores en su desarrollo; otros autores incluyen el establecimiento de validez concurrente mediante la correlación de las puntuaciones de la rúbrica con otras medidas de rendimiento de la escritura y la obtención de una indicación de fiabilidad significativa con las "técnicas clásicas" de cálculo de niveles alfa (Roblyer \& Wiencke, 2004).

Es importante analizar la validez de una rúbrica (Jonsson \& Svingby, 2007), entendida como el grado en que una rúbrica mide lo que se pretende medir (Moskal \& Leydens, 2000). Pero también lo es, dada la relevancia de este proceso, analizar la validez percibida, es decir, la que los futuros docentes otorgan mediante las valoraciones 
que realizan tras ser empleadas en evaluación de pares y autoevaluación.

El último constructo analizado es el índice Net Promoter Score (NPS), empleado para conocer el nivel de recomendación del prácticum de los futuros docentes. EI NPS consiste en un solo ítem que permite conocer la fidelidad y lealtad de los clientes ante un producto o servicio. Si los sujetos lo recomiendan (el producto o servicio) es porque solo si tienen un nivel de satisfacción alto (Reichheld, 2003). En este estudio, los clientes son los estudiantes y el servicio es el prácticum. Este indicador se evalúa en una escala consecuente que oscila entre 0 y 10 , donde 0 es muy improbable y el 10 muy probable. La persona es clasificada se según su respuesta, de 0 a 6 como "detractor", 7 u 8 " pasivo" y 9 o 10 "promotor". Con el fin de obtener el número final se resta el porcentaje de "detractores" al porcentaje de "promotores" (Grisaffe, 2007). Así, el resultado de NPS se considera bueno si supera el valor 0 y excelente si supera el 50\% (Reichheld, 2003).

\section{Metodología}

El diseño de esta investigación responde a un estudio no experimental transversal con grupos naturales de estudiantes de prácticum. En un grupo (G-experimental) la intervención consiste en la realización de varias erúbricas durante la realización de los seminarios de Prácticum semanales en la Facultad de Ciencias de la Educación de la Universidad de Granada. El Practicum I consta de 20 créditos que se cursan durante el quinto semestre, distribuidos en cuatro días a la semana en el centro escolar y un seminario semanal en la facultad. La recogida de información se basa en un sencillo cuestionario online con ítems de respuesta cerrada. En la última semana antes de finalizar el período de prácticum el cuestionario se aplica en grupos que han realizado erúbricas durante el prácticum y otros grupos en los que no (G-control). Junto a ello, todos los estudiantes realizan una e-rúbrica de autoevaluación final.

\section{Objetivos}

El presente estudio pretende conocer la opinión de los futuros docentes ante el uso de las e-rúbricas durante el prácticum, en base a las variables grado de satisfacción, utilidad, validez y recomendación. Para ello, se establecen los siguientes objetivos específicos: 


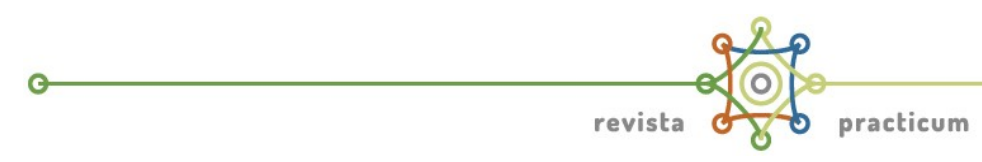

1. Identificar el grado de satisfacción con actividades realizadas en seminarios durante el prácticum, la satisfacción con el proceso evaluador basado en e-rúbricas y el índice de recomendación a otros (NPS).

2. Determinar la utilidad y validez percibida del empleo de erúbricas para la autoevaluación y reflexión del prácticum.

3. Identificar diferencias entre alumnos/as que han realizado una autoevaluación periódica del diario reflexivo a través de erúbricas y aquellos que no la han experimentado.

\section{Participantes}

La muestra del estudio está formada por 29 estudiantes, entre 20 y 26 años de edad, matriculados en el Prácticum I (2016/2017) del Grado de Educación Infantil y Grado de Educación Primaria de la Universidad de Granada (España). Se seleccionaron dos grupos de prácticum: uno experimental $(\mathrm{N}=19)$ del Grado de Educación Infantil y otro control $(\mathrm{N}=10)$ compuesto por estudiantes de ambos grados. En los resultados se denomina al grupo experimental, grupo $1 \mathrm{y}$ al grupo control, grupo 2. Se aplicó un muestreo intencional no probabilístico. Los criterios de inclusión eran: estar matriculados en el prácticum I de Educación Infantil o Primaria y, además, para el grupo experimental pertenecer al grupo seleccionado. El grupo experimental fue uno de los grupos naturales de prácticum I, donde el alumnado fue asignado aleatoriamente por la administración de la universidad. El grupo control estaba formado por estudiantes de varios grupos de prácticum I y, por tanto, con diferentes tutores académicos.

\section{Instrumento}

Para lograr los objetivos de este estudio se elaboró un cuestionario ad hoc de investigación. Los objetivos se centran en 8 ítems, distribuidos en dos partes. La primera parte contiene variables sociodemográficas de los participantes (edad, sexo, dirección de correo electrónico, nivel académico, universidad /centro educativo, calificación media (hasta ahora) en estos estudios) y una pregunta abierta para que describir las actividades realizadas en los seminarios de seguimiento del prácticum. El objetivo de dicha pregunta era conocer las actividades del alumnado ya que pertenecían a diferentes grupos de prácticum. 
La segunda parte contiene cuestiones sobre satisfacción (3 ítems), utilidad ( 2 ítems), y validez ( 2 ítems) (Tabla 1 ).

\begin{tabular}{lcc}
\hline Items cuestionario & Gr.Exp & Gr.Cont \\
\hline Indica el grado de satisfacción con actividades de seminarios de seguimiento & $\mathrm{X}$ & $\mathrm{X}$ \\
Indica el grado de satisfacción con autoevaluación en seminarios de seguimiento & $\mathrm{X}$ & - \\
Indica el grado de satisfacción con la evaluación recibida de otros colegas & $\mathrm{X}$ & \\
¿Qué utilidad tiene la autoevaluación con erúbricas? & $\mathrm{X}$ & $\mathrm{X}$ \\
¿Qué utilidad tiene la erúbrica para reflexionar sobre habilidades adquiridas durante las prácticas? & $\mathrm{X}$ & $\mathrm{X}$ \\
¿Qué validez tiene la autoevaluación con erúbricas? & & $\mathrm{X}$ \\
¿Qué validez tiene la erúbrica para autoevaluar competencias adquiridas y por adquirir? & $\mathrm{X}$ \\
Emplear la erúbrica me ha permitido involucrarme en mi aprendizaje práctico & $\mathrm{X}$ & $\mathrm{X}$ \\
\hline
\end{tabular}

Tabla 1 , ítems del cuestionario, (Elaboración propia)

Los estudiantes respondieron, según su grado de acuerdo con cada categoría, en una escala tipo Likert de 0 a 4 puntos, en la que el 0 corresponde a "no procede" y el 4 es "muy elevada". Los estudiantes de grupo control no responden a los ítems 2,3 y 8 , ya que hacen referencia al tratamiento del grupo experimental. La segunda parte finaliza con el ítem NPS (probabilidad de recomendar las prácticas a un amigo o familiar), con una opción de respuesta de 0 a 10 puntos, donde el 0 es muy improbable y el 10 muy probable, y que permite posteriormente una categorización, según su respuesta.

\section{Procedimiento}

En el mes de octubre de 2016 se entra en el campo de estudio, teniendo contacto con el alumnado y los tutores académicos. La experiencia de trabajo se llevó a cabo con los futuros docentes, a través de seminarios convocados un día a la semana (con un total de 9 sesiones). El tratamiento del grupo experimental consistió en la administración de la e-rúbrica "diario reflexivo" en autoevaluación, 
revista

evaluación de pares y evaluación del tutor en las siguientes actividades: Adaptación, Contextualización, Aspectos Psicoevolutivos y Aspectos Didácticos. Se evaluaban reflexiones extraídas de los diarios reflexivos semanales realizados durante el prácticum. Mientras que el grupo experimental empleó las e-rúbricas de autoevaluación durante el desarrollo del prácticum en cuatro ocasiones, a los demás estudiantes se le administró únicamente al final del prácticum. Las siguientes fases (tabla 2) ilustran el proceso llevado a cabo en este estudio.

\begin{tabular}{|c|c|c|c|c|}
\hline & & & $\begin{array}{l}\text { Grupo } \\
\text { experimental }\end{array}$ & $\begin{array}{l}\text { Grupo } \\
\text { control }\end{array}$ \\
\hline Fase-1 & & $\begin{array}{l}\text { Entrada en el campo. Contacto } \\
\text { estudiantes y tutores académicos }\end{array}$ & $\mathrm{X}$ & $\mathrm{X}$ \\
\hline \multirow[t]{2}{*}{ Fase-2 } & Fase-2.1 & $\begin{array}{l}\text { Aplicación e-rúbrica diario reflexivo } \\
\text { evaluación por pares }\end{array}$ & $x$ & - \\
\hline & Fase-2.2 & $\begin{array}{l}\text { Aplicación e-rúbrica diario reflexivo } \\
\text { evaluación del tutor académico }\end{array}$ & $x$ & - \\
\hline Fase-3 & & $\begin{array}{l}\text { E-rúbrica de autoevaluación del } \\
\text { aprendizaje adquirido en el prácticum }\end{array}$ & $x$ & $x$ \\
\hline Fase-4 & & Envío del cuestionario ad hoc & $x$ & $\gamma$ \\
\hline Fase-5 & & Análisis de resultados y conclusiones & $x$ & $x$ \\
\hline
\end{tabular}

Tabla 2 , fases del proceso, (Elaboración propia)

Las e-rúbricas se aplicaron mediante la herramienta federada coRubric (http://corubric.com/) de GteaVirtual (CGtea). Los estudiantes accedieron a dicha plataforma mediante su correo institucional, Facebook o Google+.

La autoevaluación se realizó mediante la e-rúbrica de autoevaluación para el aprendizaje adquirido en el prácticum (PérezTorregrosa, Gutiérrez-Santiuste \& Gallego-Arrufat, 2016), que fue creada tras una extensa revisión de literatura; validada por expertos y con una correlación intraclase de $I C C=.947$ según el acuerdo de intercodificadores. 


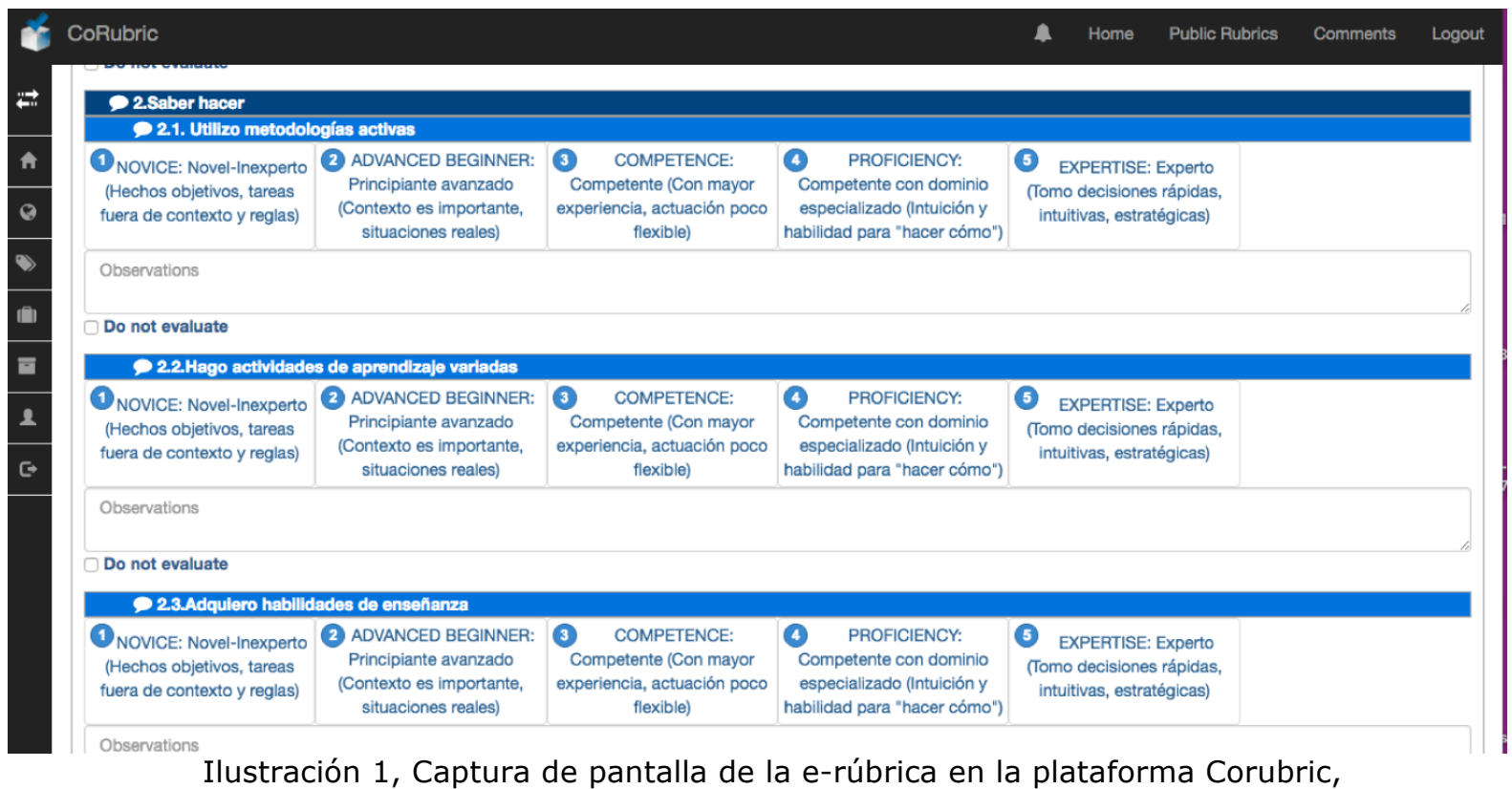

Ilustración 1, Captura de pantalla de la e-rúbrica en la plataforma Corubric,

(Elaboración propia)

Al finalizar el prácticum, la recogida de datos se llevó a cabo a través de un formulario de Google, aplicado presencialmente en estudiantes de G-exp y G-control y también remitido vía email en este último caso (recibiendo menos respuestas).-El-tratamiento estadístico de los datos se llevó a cabo mediante las hojas de cálculo de Google.

\section{Resultados}

A continuación analizamos los hallazgos obtenidos mediante el cuestionario ad hoc presentado anteriormente. Se encuentran organizados en torno a los objetivos planteados.

En cuanto al primer objetivo planteado, sobre el grado de satisfacción del proceso evaluador basado en e-rúbricas, como muestra el Gráfico 1, los resultados obtenidos sobre la variable "satisfacción" atiende a tres ítems; a) por un lado pone de relieve la elevada satisfacción del grupo control (grupo 2) ante el desarrollo de las actividades de seminario, ya que todos los valores aportados se encuentran entre 3 (elevada) y 4 (muy elevada) con un porcentaje del $90 \%$ y $10 \%$, respectivamente. Sin embargo, aunque el grupo experimental (grupo 1) presenta valores similares entre $3(68,42 \%)$ y $4(21,05 \%)$, un porcentaje menor de estudiantes señalaron la opción 1 (poco) y 2 (indiferente), con una representación del 5,26\% respectivamente; b) al igual que en el ítem anterior, el grado de satisfacción con autoevaluación en seminarios de seguimiento es mayor en el grupo control o denominado como grupo 2, pues el $70 \%$ de los estudiantes presentan una satisfacción elevada $y$, el $10 \%$ percibió una satisfacción muy elevada con la autoevaluación en 


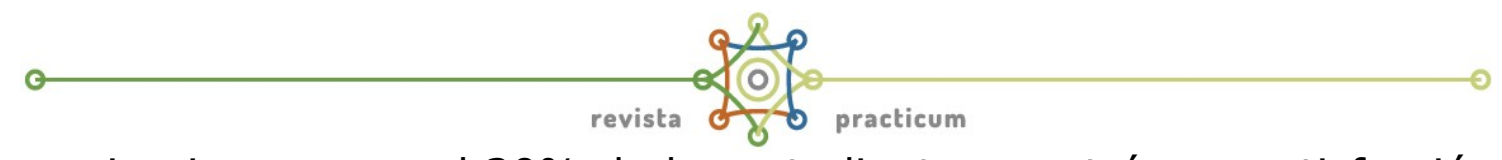

seminarios aunque el $20 \%$ de los estudiantes mostró una satisfacción indiferente. En cuanto al grupo 1, presenta una distribución de las respuestas entre 1 y 4 , donde el 5,26\% señala poca satisfacción (valor 1 ), el 26,32\% indica una satisfacción indiferente, (valor 2), el $47,37 \%$ señala elevada (valor 3 ) correspondiendo con el porcentaje más elevado, mientras que el $21,05 \%$, tenía una satisfacción muy elevada de la autoevaluación en los seminarios de seguimiento; y c) el tercer ítem, hacía referencia al grado de satisfacción presentado por la evaluación por pares, sin embargo, este ítem solo era respondido por el grupo 1 o grupo experimental, ya que trabajaban con e-rúbricas la co-evaluación o revisión por pares, con el fin de familiarizarse con dicha la herramienta. Así, los resultados obtenidos ponen de relieve la elevada satisfacción que presentan los estudiantes ante su uso, pues aportan respuestas agrupadas entre los valores 3 y 4 y, tan solo el 10,5\% mencionan una baja satisfacción.

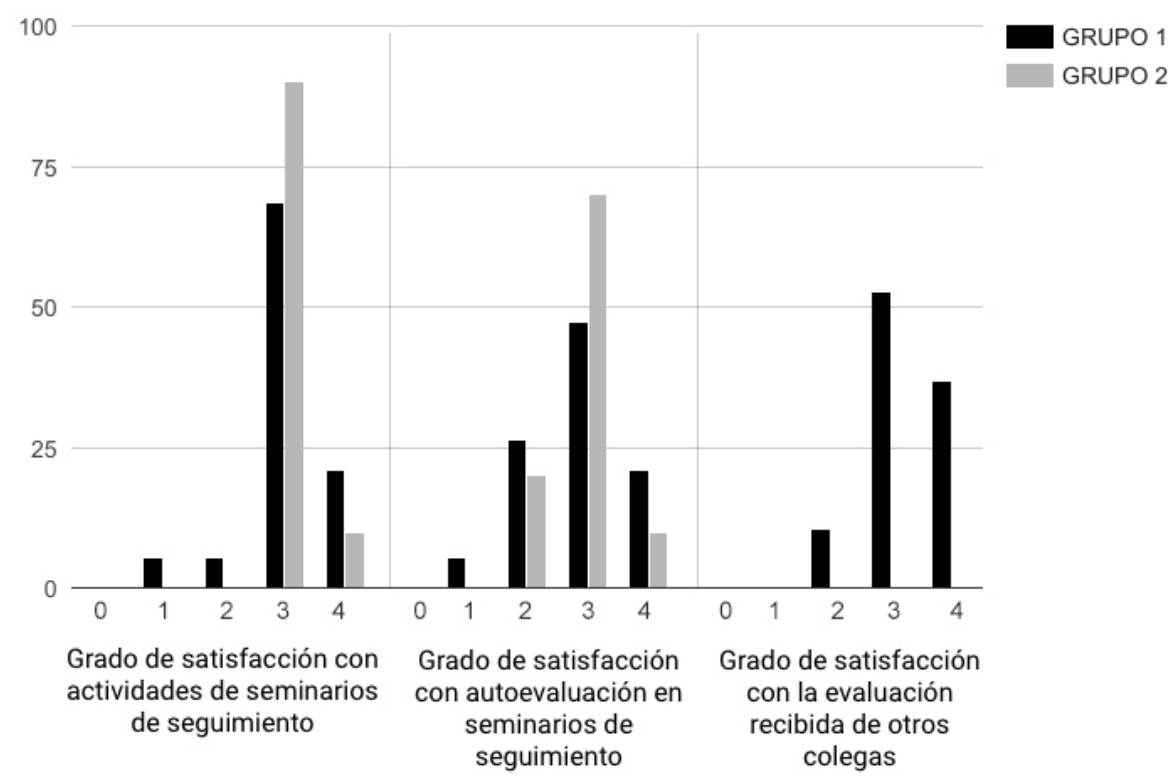

Gráfica 1, Grado de satisfacción de los estudiantes, (Elaboración propia)

Con respecto al segundo objetivo hemos podido conocer la utilidad del empleo de e-rúbricas para la autoevaluación y reflexión del prácticum. De acuerdo a la categoría "utilidad", los resultados obtenidos para cada uno de los ítems que la componen son los siguientes (ver Gráfico 2). Por un lado, ambos grupos consideran útil el uso de la e-rúbrica para la autoevaluación en un 78,95\% el grupo 1 y un $80 \%$ el grupo 2, apreciando valores muy similares. Sin 
embargo, el grupo 2 tiene mayores expectativas ante la utilidad de la e-rúbrica (mayor selección del valor 4 , con una representatividad del $20 \%$ de los estudiantes) a pesar de no estar familiarizados con la herramienta, mientras que el grupo 1 , si utilizó la e-rúbrica durante los seminarios y el $5,26 \%$ considera que es muy útil, pero encontramos un porcentaje menor de estudiantes $(15,79 \%)$ que la ha valorado como indiferente su utilidad (valor 2 ).

Por otro lado, los resultados del segundo ítem referido a la utilidad de la e-rúbrica para reflexionar sobre las habilidades adquiridas en las prácticas muestra una valoración de útil y muy útil (valores 3 y 4) para ambos grupos. La diferencia entre ellos para la valoración de "útil", es 2,63\%, sin embargo, la percepción de los estudiantes como "muy útil" (valor 4) presenta una diferencia mayor, con un $31,58 \%$ para el grupo 1 y un $50 \%$ por parte del grupo 2 .

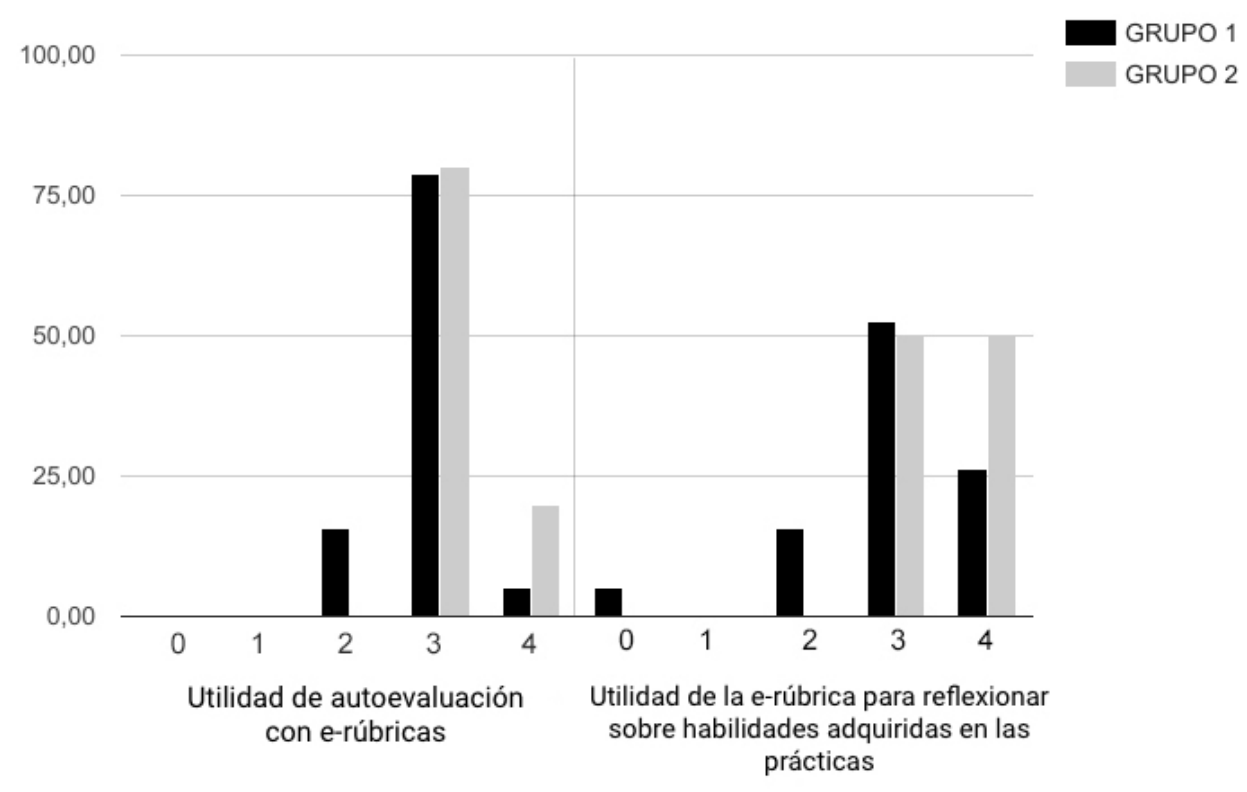

Gráfica 2, Valoración de la utilidad por los estudiantes, (Elaboración propia)

Los resultados de la categoría "validez" responden a dos ítems (Gráfico 3); a) validez de autoevaluación con e-rúbricas, donde los estudiantes de ambos grupos coinciden en sus respuestas posicionándose en el valor $3 \mathrm{y}$, por tanto, considerando válido su uso en un $73,68 \%$ para el grupo 1 y un $80 \%$ para el grupo 2 . Sin embargo, atendiendo al número de respuestas de los estudiantes 


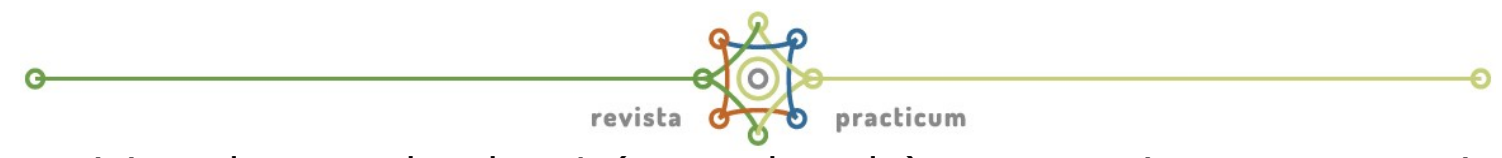

posicionados en el valor 4 (muy elevada), se aprecia un porcentaje más elevado (20\%) en el grupo 2 , que no se sometió al tratamiento y por tanto, no estaban familiarizados con la herramienta, mientras que el grupo 1 había trabajado con ella, y la valora en un 5,26\% como muy elevada su validez; y b) el ítem referido a la validez de la erúbrica para la autoevaluación de las competencias adquiridas durantes las prácticas, en el que la mayoría de los estudiantes de ambos grupos han considerado esta herramienta como elevada (valor tres) en un $57,89 \%$ para el grupo 1 y en un $70 \%$ para el grupo, apreciando entre los dos grupos una diferencia del 12,11\%. A estos datos, le siguen la respuesta ubicadas en el valor 4 , en el que los estudiantes consideran muy válida la e-rúbrica para reflexionar sobre las competencias adquiridas en el prácticum (15,79\% en el grupo 1 y $30 \%$ en el grupo 2 ). La diferencia porcentual percibida entre los datos del grupo 1 y 2 con respecto a los valores 3 y 4 , se debe una distribución de las respuestas para los valores 1 y $2(26,32 \%$ en total) por parte del grupo experimental. Recordemos que el grupo 10 experimental estaba familiarizado con la e-rúbrica.

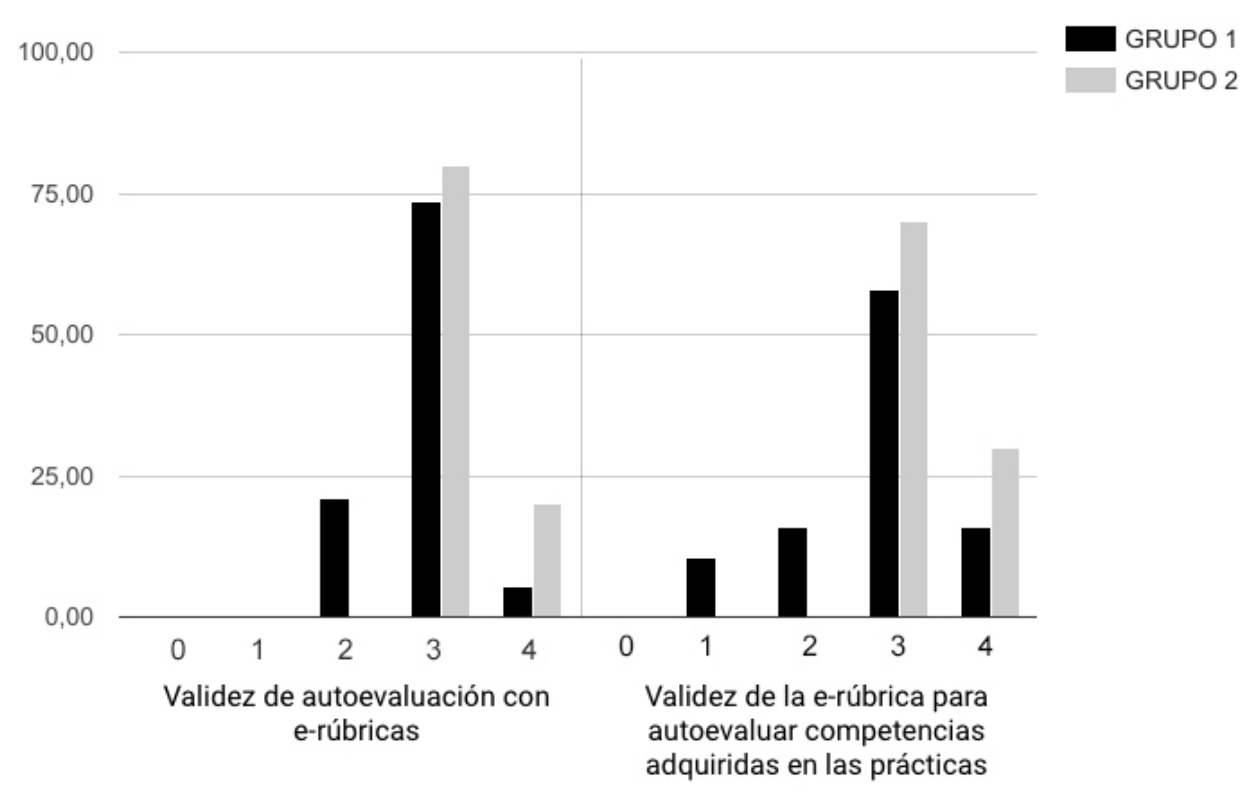

Gráfica 3, Opinión de los estudiantes sobre la validación con e-rúbricas.

(Elaboración propia)

En cuanto al análisis de las opiniones de los futuros docentes sobre la recomendación del prácticum a un amigo o familiar por el índice Net Promoter Score (NPS), encontramos que de las puntuaciones obtenidas destaca la ausencia de estudiantes 
"detractores" (valores entre 0 y 6 ), el bajo porcentaje $(6,9 \%)$ de "pasivos" (valores 7 y 8), frente a la representatividad de los estudiantes clasificados como "promotores" (los valores 9 y 10), con el $21,8 \%$ y el $71,1 \%$, obteniendo una puntuación total del $92,9 \%$. Al calcular la diferencia entre la proporción de estudiantes "promotores" y "detractores" [\%P-\%D=NPS] obtenemos un índice NPS del $93 \%$.

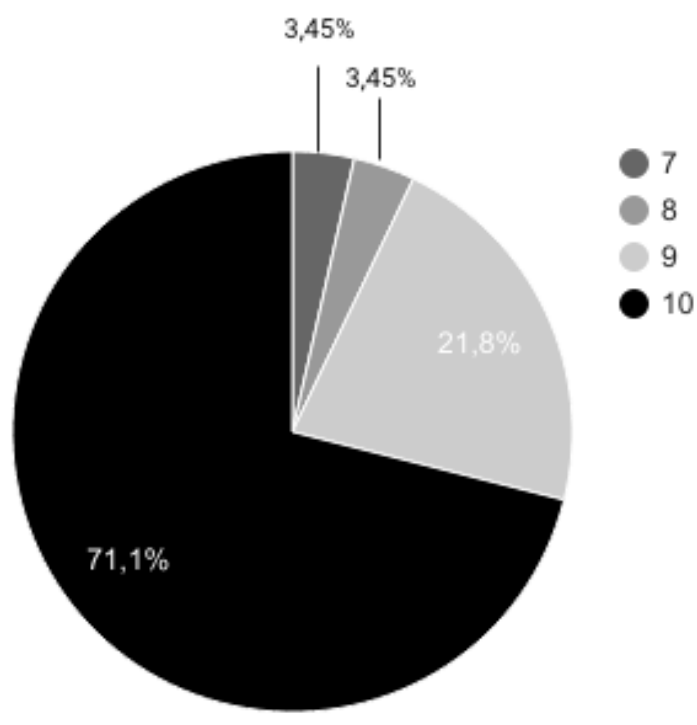

Gráfica 4, NPS de los estudiantes sobre el prácticum. (Elaboración propia)

\section{Discusión y resultados}

Tras analizar los datos se puede considerar alto el grado de satisfacción respecto al empleo de e-rúbricas para la evaluación del periodo de prácticum, según los estudiantes de los Grados de Magisterio, en consonancia con los resultados obtenidos en otros estudios al respecto como los del Panadero \& Brown, 2017. Fundamentalmente destacan la satisfacción para las actividades realizadas con e-rúbricas durante los seminarios semanales de seguimiento del periodo práctico, pues les permitió familiarizarse con la herramienta, por ejemplo la co-evaluación de los diarios reflexivos. En lo que respecta al grado de satisfacción del alumnado con la autoevaluación y coevaluación durante los seminarios de seguimiento (ítem 2) es más alto en aquellos estudiantes que han trabajado con e-rúbricas (grupo experimental) que en los que la han recibido feedback por otros medios, en los seminarios presenciales (grupo control), al igual que en los resultados obtenidos por Panadero, 
revista

Romero, \& Strijbos, 2013. Aunque los estudiantes consideran la autoevaluación, en ambos grupos, un aspecto a mejorar, pudiéndose afirmar que los estudiantes están satisfechos con la e-rúbrica para la evaluación del practicum, aunque en mayor medida para la coevaluación que para la autoevaluación, en sintonía con otros estudios realizados en el contexto español como el de Cebrián-de-laSerna, 2014 y Gallego \& Raposo, 2014 y Raposo \& Gallego, 2016.

\section{Referencias}

Andrade, H., \& Du, Y. (2005). Student perspectives on rubricreferenced assessment. Practical Assessment, Research and Evaluation, 10(3).

Andrade, H. L., Du, Y., \&., \& Mycek, K. (2010). Rubric-referenced selfassessment and middle school students' writing. Assessment in Education: Principles, Policy \& Practice, 17(2), 199-214.

Auvinen, T. (2011, November). Rubyric. In Proceedings of the 11th Koli Calling International Conference on Computing Education Research, (pp. 102-106). Koli, Filand. doi: $10.1145 / 2094131.2094152$

Atkinson, D., \& Lim, S. L. (2013). Improving assessment processes in Higher Education: Student and teacher perceptions of the effectiveness of a rubric embedded in a LMS. Australasian Journal of Educational Technology, 29(5), 651-666. doi: 10.14742/ajet.526

Boud, D., Lawson, R., \& Thompson, D. G. (2013). Does student engagement in self-assessment calibrate their judgement over time?. Assessment \& Evaluation in Higher Education, 38(8), 941-956. doi: 10.1080/02602938.2013.769198

Casaló, L. V., Flavián, C., \& Guinalíu, M. (2012). Redes sociales virtuales desarrolladas por organizaciones empresariales: antecedentes de la intención de participación del consumidor. Cuadernos de Economía y Dirección de la Empresa, 15(1), 42-51. doi: $10.1016 /$ j.cede.2011.06.003 
Cebrián-de-la-Serna, M., Serrano-Angulo, J, \& Ruiz-Torres, M. (2014). Las eRúbricas en la evaluación cooperativa del aprendizaje en la Universidad. Comunicar, 22(43), 153-161.

Davis, F. D. (1989). Perceived usefulness, perceived ease of use, and user acceptance of information technology. MIS quarterly, 13 (3), 319-340. doi: 10.2307/249008

Fry, S. A. (1990). Implementation and evaluation of peer marking in higher education. Assessment and evaluation in higher education, 15(3), 177-189. doi: 10.1080/0260293900150301

Hoepfl, M. (2007). Alternative classroom assessment tools and scoring mechanisms. Assessment of technology education: Council of technology teacher education 56th yearbook, 65-86.

Gallego-Arrufat, M. J., \& Raposo-Rivas, M. (2014). Compromiso del estudiante y percepción del proceso evaluador basado en rúbricas. REDU. Revista de Docencia Universitaria, 12(1), 197-215. doi: $10.4995 /$ redu.2014.6423

Grisaffe, D. B. (2007). Questions about the ultimate question: conceptual considerations in evaluating Reichheld's net promoter score (NPS). Journal of Consumer Satisfaction, Dissatisfaction and Complaining Behavior, 20, 36-53.

Jonsson, A., \& Svingby, G. (2007). The use of scoring rubrics: Reliability, validity and educational consequences. Educational research review, 2(2), 130-144. doi: 10.1016/j.edurev.2007.05.002

Legris, P., Ingham, J., \& Collerette, P. (2003). Why do people use information technology? A critical review of the technology acceptance model. Information \& management, 40(3), 191-204. doi: 10.1016/S0378-7206(01)00143-4

Moskal, B. M., \& Leydens, J. A. (2000). Scoring rubric development: Validity and reliability. Practical assessment, research \& evaluation, 7(10), 71-81.

Padilla Meléndez, A., \& Garrido Moreno, A. (2006). El uso de tecnologías basadas en Internet para el aprendizaje: Un estudio 
revista

exploratorio en el contexto del modelo de aceptación de la tecnología. Investigaciones europeas de dirección de la empresa (IEDEE), 12(2), 217-230.

Panadero, E., Romero, M., \& Strijbos, J. W. (2013). The impact of a rubric and friendship on peer assessment: Effects on construct validity, performance, and perceptions of fairness and comfort. Studies in Educational Evaluation, 39(4), 195-203. doi:10.1016/j.stueduc.2013.10.005

Panadero, E., \& Romero, M. (2014). To rubric or not to rubric? The effects of self-assessment on self-regulation, performance and selfefficacy. Assessment in Education: Principles, Policy \& Practice, 21(2), 133-148. doi:10.1080/0969594X.2013.877872

Panadero, E., \& Brown, G. T. (2017). Teachers' reasons for using peer assessment: positive experience predicts use. European Journal of Psychology of Education, 32(1), 133-156.

Park, S. Y. (2009). An analysis of the technology acceptance model in understanding university students' behavioral intention to use elearning. Educational technology \& society, 12(3), 150-162.

Pérez-Torregrosa, A.B.,Gutiérrez-Santiuste, E., \& Gallego-Arrufat, M.J. (2016, september). Autoevaluación con e-rúbrica en practicum: construcción y validación del instrumento. En Pedro, N., Pedro, A., Filipe Matos, J., Piedade, J., \& Fonte, M. (Eds.), Digital Technologies \& Future School Atas do IV Congresso Internacional TIC e Educação 2016 (pp. 387-393). Lisboa: Instituto de Educação da Universidade de Lisboa.

Perrone, M. y Propper, F. (Eds.). (2007). Satisfacción. En Perrone Diccionario de educación. pp.339-340. Buenos Aires: Alfagrama.

Prins, F. J., Sluijsmans, D. M., Kirschner, P. A., \& Strijbos, J. W. (2005). Formative peer assessment in a CSCL environment: A case study. Assessment \& Evaluation in Higher Education, 30(4), 417-444. doi: $10.1080 / 02602930500099219$ 
Raposo-Rivas, M., \& Gallego-Arrufat, M. J. (2016). University Students' Perceptions of Electronic Rubic-Based Assessment. Digital Education Review, (30), 220-233.

Reddy, Y. M., \& Andrade, H. (2010). A review of rubric use in higher education. Assessment \& evaluation in higher education, 35(4), 435448. doi:10.1080/02602930902862859

Reichheld, F. F. (2003). The one number you need to grow. Harvard business review, 81(12), 46-55.

Rezaei, A. R., \& Lovorn, M. (2010). Reliability and validity of rubrics for assessment through writing. Assessing writing, 15(1), 18-39. doi: 10.1016/j.asw.2010.01.003

Roblyer, M. D., \& Wiencke, W. R. (2004). Exploring the interaction equation: Validating a rubric to assess and encourage interaction in distance courses. Journal of Asynchronous Learning Networks, 8(4), 24-37.

Sánchez, M. J., Martín, F. A., \& Villarejo, F. (2016). El modelo TAM y la enseñanza superior. Una investigación del efecto moderador del sexo. Revista Española de Pedagogía, 238, 459- 478.

Selim, H. M. (2003). An empirical investigation of student acceptance of course websites. Computers \& Education, 40(4), 343-360. doi: $10.1016 /$ S0360-1315(02)00142-2

Serrano Angulo, J., \& Cebrián Robles, D. (2014). Usabilidad y Satisfacción de la e-Rúbrica. Revista de Docencia Universitaria, 12(1), 177-195.

Topping, K. J., Smith, E. F., Swanson, I., \& Elliot, A. (2000). Formative peer assessment of academic writing between postgraduate students. Assessment \& Evaluation in Higher Education, 25(2), 149-169. doi: 10.1080/713611428

Teo, T., Lee, C. B., Chai, C. S., \& Wong, S. L. (2009). Assessing the intention to use technology among pre-service teachers in Singapore 
and Malaysia: A multigroup invariance analysis of the Technology Acceptance Model (TAM). Computers \& Education, 53(3), 1000-1009. doi:10.1016/j.compedu.2009.05.017 